\title{
Programa de Mentoria da Faculdade de Medicina da Universidade Federal de Alfenas: relato de experiência
}

\author{
Mentoring Program at the Medical School of the Federal University of Alfenas: experience report \\ Dyecika Souza Couto' (D)|dyecikacouto99@gmail.com \\ Gabriela Itagiba Aguiar Vieira' (D) gabrielavieiramfc@gmail.com \\ Stela Lima Mulati' 1 (D) stelalima_20@hotmail.com \\ Vânia Regina Bressan' (D) vania.bressan@unifal-mg.edu.br
}

\section{RESUMO}

Introdução: O Programa de Mentoria do curso de Medicina da Universidade Federal de Alfenas (Unifal) foi inspirado em programas de mentoring implantados em escolas médicas brasileiras desde a década de 1990, reconhecendo que a formação do futuro médico é marcada por intenso estresse acadêmico e emocional.

Relato de experiência: O Programa de Mentoria tem papel preventivo e de suporte para o desenvolvimento pessoal e profissional dos estudantes de Medicina. As ações visam acompanhar o estudante longitudinalmente para proporcionar momentos de reflexão, tomada de consciência e desenvolvimento de competências para enfrentar os meios acadêmico, social e profissional. O mentor provê ao estudante uma figura-modelo que atua como suporte para auxiliá-lo nas vicissitudes do processo de ser médico.

Discussão: Segundo os participantes, o Programa de Mentoria permite troca de experiências, aprendizagem e reflexão sobre temas relevantes da profissão e da vida acadêmica, atendendo às exigências das Diretrizes Curriculares Nacionais para os Cursos de Medicina para uma formação geral, humanista, crítica, reflexiva e ética.

Conclusão: O Programa de Mentoria é exemplo de intervenção possível para prevenção de doenças e promoção da saúde durante a formação médica e contribui para o desenvolvimento de habilidades nos contextos pessoal e profissional.

Palavras-chave: Educação Médica; Mentoria; Estudante.

\section{ABSTRACT}

Introduction: The Mentoring Program of the Medical School at UNIFAL-MG was inspired by Mentoring Programs implemented in Medical Schools in Brazil since the 1990s, recognizing that training to be a physician is marked by intense academic and emotional stress.

Experience report: The Mentoring Program has a preventive and supportive role for the personal and professional development of medical students. The actions aim to accompany the student longitudinally to provide moments of reflection, awareness, and the skills to face the academic, social and professional environment. The mentor represents a supportive model figure for the student, to help him overcome the difficulties in the process of being a physician.

Discussion: According to the participants, the Mentoring Program allows the exchange of experiences, learning and reflection on relevant topics of the profession and academic life, meeting the requirements of the National Curriculum Guidelines for Medical Courses for general, humanistic, critical, reflective and ethical training.

Conclusion: The Mentoring Program exemplifies a method of intervention for prevention and health promotion during medical training and contributes to the development of skills in both a personal and professional setting.

Keywords: Medical Education; Mentoring; Student.

${ }^{1}$ Universidade Federal de Alfenas, Alfenas, Minas Gerais, Brasil.

Editora: Lia Marcia Cruz da Silveira

Recebido em 03/03/21; Aceito em 03/05/21.

Avaliado pelo processo de double blind review. 


\section{INTRODUÇÃO}

Não há dúvida de que o ingresso na universidade representa uma conquista, no entanto pode se tornar uma fase crítica de maior vulnerabilidade para o início e a manutenção do uso de álcool e outras drogas, e o desenvolvimento de sofrimento e transtornos psíquicos, pois é um período em que o jovem se distancia do núcleo familiar e se insere em um novo contexto que requer adaptações, novas posturas e responsabilidades. Sabe-se que a prevalência de sintomas depressivos, ansiedade, uso de álcool e outras drogas e suicídio é maior entre os estudantes de Medicina quando comparada à população geral. Isso ocorre porque esses estudantes estão sujeitos a potentes estressores, tais como sobrecarga de conhecimentos, competição, dificuldade na administração do tempo, individualismo, responsabilidade e expectativas sociais do papel de médico, contato com a morte e processos patológicos, exame físico de pacientes, medo de adquirir doenças, de cometer erros, sentimento de impotência diante de certas doenças, além de rede de apoio deficiente1. Por isso, fazem-se necessárias propostas de prevenção (apoio ou suporte) e intervenção para essa população. Nesse sentido, os programas de mentoria têm papel preventivo e de suporte para o desenvolvimento pessoal e profissional dos estudantes de Medicina ${ }^{1,2}$.

As ações dos programas de mentoria visam acompanhar o estudante longitudinalmente em um ambiente humanizado para proporcionar momentos de reflexão, tomada de consciência e desenvolvimento de competências para enfrentar os meios acadêmico, social e profissional. Assim, eles têm como objetivo acolher o estudante que sai de seu núcleo familiar e se insere no ambiente da universidade, com exigências de novas posturas e responsabilidades. Além disso, tencionam oferecer suporte para que o estudante enfrente a formação acadêmica e a profissão com menos angústia e adoecimento, e promover discussões e reflexões sobre temas de interesse dos estudantes, a fim de auxiliá-los no desenvolvimento de competências para enfrentar dificuldades pessoais, acadêmicas e profissionais. Por meio de suas ações e da proximidade nos pequenos grupos, é possível detectar estudantes em sofrimento psíquico ou que possam apresentar quadros de transtornos mentais ou comportamentais relacionados ao uso de álcool e outras substâncias psicoativas para que possam ser encaminhados para o tratamento ${ }^{3}$.

Segundo as propostas dos programas de mentoria, um mentor acompanha longitudinalmente um grupo de estudantes. O mentor é considerado figura que inspira, dá conselhos e auxilia o estudante a atravessar a jornada do curso de Medicina, sem assumir a postura de avaliador, o papel de pai, amigo ou psicoterapeuta. Um mentor pode ocupar um espaço social único para o jovem em busca de si mesmo, além de proporcionar oportunidades para que os estudantes concretizem os seus objetivos pessoais e profissionais preservando a saúde física e mental, de modo a exercer, ainda melhor, os cuidados da medicina. As características consideradas importantes para ser um mentor são: estar inserido no contexto da graduação, ter postura empática, bom relacionamento com os estudantes, capacidade para ouvir, disponibilidade dentro e fora do grupo, paciência e abertura para compartilhar experiências ${ }^{3}$. Este relato apresenta a experiência da implantação e do desenvolvimento do Programa de Mentoria da Faculdade de Medicina da Universidade Federal de Alfenas (Unifal), de Minas Gerais, uma instituição pública centenária, na qual o curso de Medicina foi iniciado em 2014. A instituição oferta 60 vagas com acesso anual pelo Sistema de Seleção Unificada (Sisu).

\section{RELATO DE EXPERIÊNCIA}

O Programa de Mentoria surgiu da necessidade do curso de Medicina da Unifal de adotar uma estrutura que respondesse às exigências das Diretrizes Curriculares Nacionais para os Cursos de Medicina em relação à criação de um Núcleo de Apoio ao Estudante. Assim, a coordenação do curso com o apoio do Núcleo Docente Estruturante indicou uma técnica administrativa em educação, com formação em saúde mental, para o planejamento e a implantação do Programa de Mentoria. O Programa de Mentoria foi inspirado em programas de mentoring que têm sido implantados em escolas médicas brasileiras desde a década de 1990, reconhecendo que a formação do futuro médico é marcada por intenso estresse acadêmico e emocional ${ }^{1-3}$.

Em 2016,o Programa de Mentoria começou suas atividades. Ao início das aulas, logo após a apresentação da proposta, os estudantes foram convidados a responder a um questionário no qual deveriam indicar os professores que gostariam de ter como mentores e os que não gostariam, assim como os temas que desejavam discutir ao longo das reuniões, as expectativas e o interesse em participar do Programa de Mentoria.

Em paralelo, apresentou-se a proposta do programa aos 28 docentes do curso de Medicina que foram convidados, pessoalmente, a participar da capacitação em mentoria. Todos os docentes participaram da capacitação de mentores que foi realizada na própria universidade, com exposições dialogadas, vivências grupais e discussões, com duração de 20 horas. Os temas abordados foram: mentoria/mentoring como estratégia de apoio ao estudante; Centro de Atenção Psicossocial: serviço de saúde mental do município; serviços e estratégias de apoio institucional; (re)conhecendo a situação de saúde mental e sofrimento psíquico do estudante universitário na 
contemporaneidade; como identificar sintomas de ansiedade, depressão e transtornos de personalidade; como ajudar um estudante em risco de suicídio; uso de álcool e outras drogas: prevenção, riscos e como ajudar; discriminação racial, de gênero e religiosa; a importância da espiritualidade na prevenção de transtornos mentais; a relação professorestudante na educação superior; como utilizar a abordagem centrada na pessoa na relação professor-estudante; dinâmicas de grupo/abordagens lúdicas em mentoria. A capacitação foi importante para que os docentes desenvolvessem habilidades para identificar sintomas ou situações risco, oferecer suporte e realizar encaminhamentos, se necessário, aos serviços da instituição e/ou fora dela.

Entre os docentes que participaram da capacitação, todos aderiram ao programa, entretanto sete não foram indicados pelos estudantes como mentores e, por isso, não assumiram grupos de mentoria. Cada mentor acolheu um grupo formado por meio de sorteio, incluindo estudantes de diferentes períodos para facilitar e enriquecer a troca de experiências entre eles. A proposta é um acompanhamento longitudinal ao longo de todo o curso, e os membros continuam no mesmo grupo enquanto desejarem. Ao início de cada semestre, os grupos são atualizados a partir da manifestação de interesse em continuar no programa ou sair dele, e, com a saída de um membro, outro estudante pode ocupar a vaga de forma a manter o grupo com até dez mentorados. Os estudantes podem escolher outro mentor de acordo com a afinidade ou trocar de grupo caso manifestem interesse. O mentorado que deixa o Programa de Mentoria pode retornar a qualquer momento, desde que manifeste o interesse. O mentor é desligado do programa caso não realize as reuniões mensais com o seu grupo e/ou não participe das reuniões mensais de supervisão por um período de seis meses.

As reuniões de mentoria são agendadas em horário conveniente para os integrantes do grupo e podem durar até duas horas. São realizadas dentro ou fora da instituição, conforme o combinado entre os membros, desde que favoreçam o processo de diálogo e as trocas de experiências, e que se garanta o sigilo. Ao final de cada reunião, o mentor realiza um breve registro com as atividades desenvolvidas, os resultados e os participantes.

A coordenação do Programa de Mentoria promove reuniões de supervisão mensais com a participação dos mentores. Nas reuniões de supervisão, ocorrem discussões e orientação, e um profissional especialista é convidado a discorrer ou discutir com o grupo de mentores sobre temáticas importantes para a formação pessoal e profissional do estudante de Medicina. As reuniões de supervisão são de extrema importância para o andamento do projeto, pois, por meio delas, a coordenação discute casos de estudantes que necessitam de encaminhamento a profissionais (psicólogo, psiquiatra e/ou outros), dificuldades de manejo do grupo e outras questões de interesse do mentor. Além disso, as reuniões de supervisão constituem espaço para a mentoria por pares entre os próprios mentores que aproveitam a oportunidade para expressar seus sentimentos e suas inquietações com confiança e segurança.

Entre 2016 e 2018, o Programa de Mentoria foi registrado como atividade de extensão e, a partir de 2019, passou a ser registrado pela Pró-Reitoria de Graduação. A participação é voluntária e estimulada por meio de horas de atividades complementares, na condição de os estudantes apresentarem $75 \%$ de frequência nos encontros realizados.

Semestralmente, estudantes e mentores respondem ao questionário de avaliação do Programa de Mentoria. Desse modo, a mentoria é monitorada e avaliada por meio de relatórios dos encontros e das reuniões de supervisão, registro de frequência e questionário semestral. No questionário de avaliação do Programa de Mentoria, os participantes relataram que o programa permite troca de experiências, aprendizagem e reflexão sobre temas relevantes da profissão e da vida acadêmica. Além disso, os estudantes referem que o programa permite maior proximidade com os professores e que assim se sentem acolhidos. Contudo, destacaram a dificuldade em expressar aspectos pessoais no grupo e em agendar os encontros em horário comum a todos os integrantes, faltas frequentes de colegas, a falta de compromisso de alguns mentores e a formação de pequenos grupos dentro de grupos do mentoring. No segundo ano de atividade, o Programa de Mentoria foi contemplado com uma bolsa de extensão para um estudante que promovesse a comunicação entre os grupos, entre os mentores e entre os participantes e a coordenação, e que auxiliasse na divulgação do programa, na acolhida aos novos membros, nos registros dos encontros e na sistematização dos dados gerados pelo programa. Isso foi possível graças ao reconhecimento dos diversos benefícios que o Programa de Mentoria tem proporcionado aos estudantes de Medicina, buscando, além da prevenção de doenças e promoção do bem-estar durante a formação médica, a contribuição para uma formação geral, humanista, crítica, reflexiva e ética. Esse estudante bolsista foi de extrema importância para a interlocução entre coordenação e estudantes de Medicina para o aprimoramento e desenvolvimento do Programa de Mentoria.

A avaliação do Programa Mentoria indicou feedback positivo de estudantes e docentes, e, em outubro de 2019, o regulamento foi alterado para atender estudantes de outros cursos, além de Medicina. Por isso, o programa promoveu capacitação cruzada entre mentores "seniores" por meio de atividades de desenvolvimento docente com a apresentação e discussão de estratégias de apoio aos estudantes, com o objetivo de captar mentores para os outros cursos. Atualmente 
Tabela 1. Relação de mentorados e grupos de mentoria de acordo com o ano.

\begin{tabular}{ccc}
\hline Ano & Número de mentorados & Número de grupos \\
\hline 2016 & 113 & 17 \\
2017 & 153 & 17 \\
2018 & 125 & 13 \\
2019 & 140 & 14 \\
2020 & 173 & 18 \\
\hline
\end{tabular}

há grupos de mentoria nos cursos de Medicina, Enfermagem, Biologia e Nutrição. No curso de Medicina, há seis grupos com a participação de 60 estudantes.

Diante do distanciamento social imposto pela pandemia da coronavirus disease 2019 (Covid-19), desde março de 2020, e apesar de alguns considerarem os encontros virtuais um fator dificultador para a adesão ao Programa de Mentoria, diversos grupos continuaram se encontrando por meio de plataformas para reuniões on-line. Para esses grupos, a adesão à forma virtual foi surpreendente com a participação de um maior número de mentorados quando comparado às reuniões presenciais, de modo que se pode inferir que a conversa e o suporte são imprescindíveis para o enfrentamento de tempos tão difíceis.

Segundo os estudantes, o Programa de Mentoria contribui para o desenvolvimento de habilidades de escuta qualificada, empatia, comunicação e acolhimento. Entre os fatores que influenciam negativamente a adesão ao Programa de Mentoria, destacam-se o excesso de atividades que desenvolvem na universidade e a falta de tempo.

\section{DISCUSSÃO}

O Programa de Mentoria é proposto aos estudantes de Medicina como estratégia para oferecer suporte pessoal diante do intenso estresse acadêmico e emocional ${ }^{4}$, além de estimular o desenvolvimento profissional ${ }^{5}$. A experiência de implantação e desenvolvimento do Programa de Mentoria revela a percepção de benefícios para mentores, estudantes, organização e sociedade. Entretanto, diversos desafios são encontrados como barreiras para a sua continuidade ${ }^{6}$.

Esses desafios estão relacionados às características intrínsecas do estudante de Medicina e às do ambiente em que ele está inserido. Alguns autores apontam como características intrínsecas dos estudantes a preocupação quanto à privacidade, as dificuldades de tempo e acesso aos recursos, a resistência em reconhecerem que necessitam de ajuda e a tendência a minimizarem os sintomas ${ }^{7-10}$.

A adesão desafia os programas pioneiros, assim como o nosso em que a adesão é decrescente e preocupa a coordenação e frustra mentores e mentorados. Segundo a pesquisa realizada por Bellodi et al. ${ }^{11}$, assim como no Programa de Mentoria da
Unifal, a falta de tempo e o excesso de atividades são apontados como fatores que influenciam negativamente a adesão de estudantes e mentores.

O estudo de Bellodi et al. ${ }^{11}$ também evidenciou uma dificuldade enfrentada pelo nosso grupo: a criação de um espaço seguro para que os estudantes possam se sentir confortáveis para dividir seus sentimentos e pensamentos, pois a falta de um local destinado a esse fim impede o entrosamento efetivo do grupo. A criação de um ambiente acolhedor e seguro é essencial para aumentar a adesão e favorecer o vínculo entre os mentorados.

Para Martins et al. ${ }^{12}$, a falta de compreensão sobre o que é o mentoring e como ele pode auxiliar na formação médica e no enfrentamento das dificuldades influencia negativamente a adesão ao Programa de Mentoria. Isso reforça a necessidade de divulgação e orientação sobre objetivos e justificativas do Programa de Mentoria, especialmente entre os recémchegados à universidade ${ }^{12}$, assim como é realizado na Unifal.

Horário protegido, disciplina obrigatória, atividades complementares, não há consenso sobre a melhor solução, e explorar as razões dos participantes pode contribuir para o reconhecimento da melhor estratégia para favorecer a adesão ${ }^{11}$. Quanto aos mentores, a satisfação por participar do Programa de Mentoria é inquestionável, mas, além disso, deve-se pensar em estratégias que motivem ou recompensem a participação deles.

Treinamento de mentores, supervisão periódica e avaliação semestral favorecem a continuidade e o aprimoramento do programa ${ }^{11}$, e, segundo Singh et al. $^{13}$, o sucesso do mentoring depende do preparo adequado dos mentores. Nesse sentido, a avaliação positiva do Programa de Mentoria da Unifal pode se dever, entre outros fatores, à capacitação dos mentores e à supervisão periódica, assim como sugerido pelos autores que encorajam a participação dos mentores nos programas anuais de treinamento em mentoria ${ }^{14}$.

Assim como o estudo de Mustafá et al. ${ }^{4}$ revelou a importância da mentoria para a formação de estudantes de Medicina, Enfermagem, Farmácia e Odontologia, ressalta-se a ampliação do Programa de Mentoria para outros cursos como uma conquista, e esperamos que contribua para a formação integral e humana dos estudantes de todos os cursos oferecidos pela Unifal.

Apesar das críticas ao e-mentoring, em tempos de pandemia e isolamento social, as reuniões on-line supriram a necessidade de conversa e suporte para enfrentar tempos tão difíceis e justificaram a manutenção das atividades de forma remota ${ }^{15}$.

\section{CONCLUSÃO}

A experiência do Programa de Mentoria da Unifal demonstra que o mentoring é exemplo de intervenção possível 
para prevenção de doenças e promoção da saúde durante a formação médica, e contribui para o desenvolvimento de habilidades destinadas ao aperfeiçoamento pessoal e profissional.

\section{CONTRIBUIÇÃO DAS AUTORAS}

Todas as autoras participaram do planejamento da pesquisa, da coleta e análise dos dados, e da redação do artigo.

\section{CONFLITO DE INTERESSES}

Declaramos não haver conflito de interesses.

\section{FINANCIAMENTO}

Declaramos não haver financiamento.

\section{REFERÊNCIAS}

1. Bellodi PL, Martinho T, Massaroppe B, Martins MA, Santos MAS. Temas para um programa de tutoria em medicina: uma investigação das necessidades dos alunos da FMUSP. Rev Bras Educ Med. 2004;28(2):119-27.

2. Costa BEP. Mentoring na Faculdade de Medicina. Sci Med. 2008;18(4):152-3.

3. Gonçalves MCN, Bellodi PL. Ser mentor em medicina: uma visão arquetípica das motivações e transformações na jornada. Interface (Botucatu). 2012;16(41):501-14.

4. Mustafá AMM, Gomides MM, Costa JL, Pires AT, Carvalho IGM. Mentoring e educação em saúde. J BusinessTechn. 2019;9(1):147-53.
5. Botti SH, Rego S. Perceptor, supervisor, tutor e mentor: quais são seus papéis? Rev Bras Educ Med. 2008;32(3):363-73.

6. Flash DH, Smith MF, Smith WG, Glasser ML. Faculty mentors for medical students. J Med Educ. 1982;57(1):514-20.

7. Spencer J. Physician, heal thyself: but not on your own, please. Med Educ. 2005;39(1):548-9.

8. Chew-Graham CA, Rogers A, Yassin N."I wouldn't want it on my CV or their records": medical students' experiences of help-seeking for mental health problems. Med Educ. 2003;37(1):873-80.

9. Roberts LW, Warner TD, Carter D, Frank E, Ganzini L, Lyketsos C. Caring for medical students as patients: access to services and care-seeking practices of 1,027 students at nine medical schools. Acad Med. 2000;75(3):272-7.

10. Hooper C, Meakin R, Jones M. Where students go when they are ill: how medical students access health care. Med Educ. 2005;39(1):588-93.

11. Bellodi PL, Chebabo R, Abensur SI, Martins MA. Mentoring: ir ou não ir, eis a questão: um estudo qualitativo. Rev Bras Educ Med. 2011;35(2):237-45.

12. Martins AF, Bellodi PL. Mentoring: uma vivência de humanização e desenvolvimento no curso médico. Interface. 2016;20(58):715-26.

13. Singh S, Singh N, Dhaliwal U. Near-peer mentoring to complement faculty of first-year medical students in India. J Educ Eval Health Prof. 2014;11(12):1-7.

14. Taherian K, Shekarchian M. Mentoring for doctors. Do its benefits outweigh its disadvantages? Med Teach. 2008;30(4):95-9.

15. Frei E, Stamm M, Buddeberg-Fischer B. Mentoring programs for medical students: a review of the PubMed literature 2000-2008. BMC Med. Educ. 2010;32(1):1-14. 